EPJ Web of Conferences 116, 04004 (2016)

DOI: $10.1051 /$ epjconf/201611604004

(C) Owned by the authors, published by EDP Sciences, 2016

\title{
Low-energy point source searches with IceCube
}

\author{
Sebastian Euler ${ }^{1, \mathrm{a}}$, David Altmann ${ }^{2,3, \mathrm{~b}}$, Rickard Ström ${ }^{1, \mathrm{c}}$ for the IceCube Collaboration ${ }^{\mathrm{d}}$ \\ ${ }^{1}$ Department of Physics and Astronomy, Uppsala University, 75120 Uppsala, Sweden \\ ${ }^{2}$ Deutsches Elektronen-Synchrotron (DESY), 15735 Zeuthen, Germany \\ ${ }^{3}$ Erlangen Centre for Astroparticle Physics (ECAP), Friedrich-Alexander-Universität \\ Erlangen-Nürnberg, 91058 Erlangen, Germany
}

\begin{abstract}
Due to the overwhelming background of atmospheric muons, the traditional IceCube point source search in the Southern Hemisphere is mainly sensitive to neutrinos with energies above $100 \mathrm{TeV}$. A new approach focuses on events starting inside the instrumented volume. By utilizing different veto techniques we are able to significantly reduce the energy threshold and can now for the first time explore the entire Southern Hemisphere at neutrino energies as low as $100 \mathrm{GeV}$. We present the results of two analyses targeting slightly different energy ranges. Both use one year of data taken with the completed IceCube detector in 2011/12.
\end{abstract}

\section{Introduction}

The standard IceCube neutrino point source search [1] is most sensitive in the Northern Hemisphere, where the Earth shields the massive background from atmospheric muons. In the Southern Hemisphere, this analysis is limited to the highest energies above $100 \mathrm{TeV}$, where the atmospheric background is sufficiently low. The Southern Hemisphere is, however, an interesting target, in particular at lower energies, as it contains the Galactic center and a large part of the inner Galaxy, with many potential neutrino sources $[2,3]$.

Events starting inside the IceCube detector [4] are an unambiguous signature of a neutrino interaction. By utilizing different veto techniques and focusing on starting events it is possible to reduce the background to a tolerable level and thus significantly lower the energy threshold for neutrino point source searches in the Southern Hemisphere. Here we present two such analyses, one aimed at an energy range of a few $\mathrm{TeV}$ to $100 \mathrm{TeV}$ (STeVE - Starting TeV Events), and the other one aimed at even lower energies above 100GeV (LESE - Low Energy Starting Events).

\footnotetext{
a e-mail: sebastian.euler@icecube.wisc.edu

be-mail: david.altmann@icecube.wisc.edu

ce-mail: rickard.strom@icecube.wisc.edu

$\mathrm{d}_{\text {http }}$ ///wWw. icecube.wisc.edu
}

This is an Open Access article distributed under the terms of the Creative Commons Attribution License 4.0, which permits unrestricted use, distribution, and reproduction in any medium, provided the original work is properly cited. 


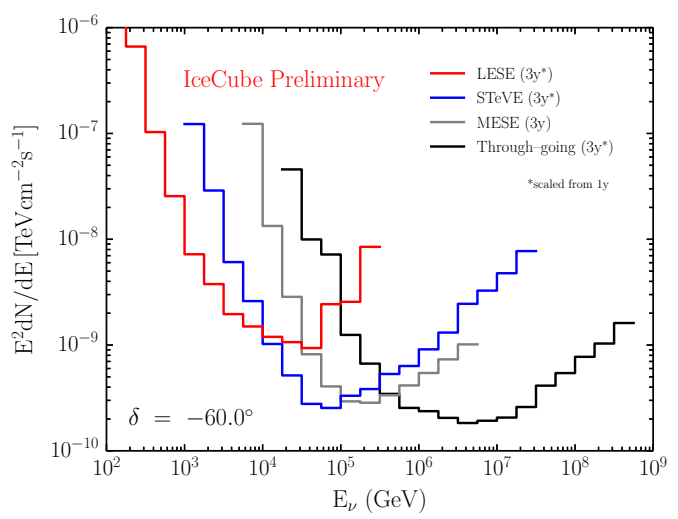

Figure 1. Differential sensitivities of four IceCube point source searches $[1,6]$ targeting different energy ranges, corresponding to 3 years of operation.

\section{Data samples}

Both analyses presented here use data from the first year of the completed 86-string IceCube detector. Due to slightly different run selections, there is a small difference in the final livetimes, 329days for LESE and 337days for STeVE. Both analyses rely on veto methods to reject the atmospheric muon background, using for example timing information of hits in the outer layers of IceCube and in IceTop. A cornerstone of both selections is the use of the FiniteReco algorithm [5], which reconstructs the neutrino interaction vertex based on the hit pattern seen in the detector. Events with a vertex outside the detector are rejected. For the final step of the event selection both analyses employ Boosted Decision Trees (BDT). The final LESE sample contains 6191 events and the STeVE sample 10178 events, with both samples still being dominated by atmospheric muons leaking in through the veto and mimicking starting neutrinos. The median angular resolution of both selections is about $2^{\circ}$, slightly worse than for the standard IceCube point source search, due to the lower energies of the events and the resulting shorter track lengths. Figure 1 shows the differential sensitivities of IceCube analyses aimed at different energy ranges: the LESE and STeVE analyses at the lowest energies, the MESE (Medium Energy Starting Events) analysis at somewhat higher energies [6] and the standard point source search (labeled "Through-going") at the highest energies [1]. Together, these analyses cover an energy range of 7 orders of magnitude from $100 \mathrm{GeV}$ to the EeV-range. Figure 2 shows the effective areas for the same set of analyses, compared to an analysis by ANTARES [7].

\section{Analysis method}

Both the LESE and the STeVE analysis use an unbinned maximum likelihood method [8] to search for a spatial clustering of events. The full likelhood $\mathcal{L}$ is given by

$$
\mathcal{L}\left(n_{s}, \gamma\right)=\prod_{i}^{N}\left(\frac{n_{s}}{N} S_{i}(\Delta \psi, \sigma, E ; \gamma)+\left(1-\frac{n_{s}}{N}\right) B_{i}(\delta, E)\right)
$$

For a given event and position in the sky we evaluate the compatibility with being signal or background, using the PDFs $S_{i}$ and $B_{i}$. The signal PDF $S_{i}$ depends on the angular difference $\Delta \psi$ between the reconstructed event direction and the considered source position, the angular uncertainty gma and reconstructed energy $E$ of the event, and the spectral index of the source $\gamma$. The background PDF $B_{i}$ 


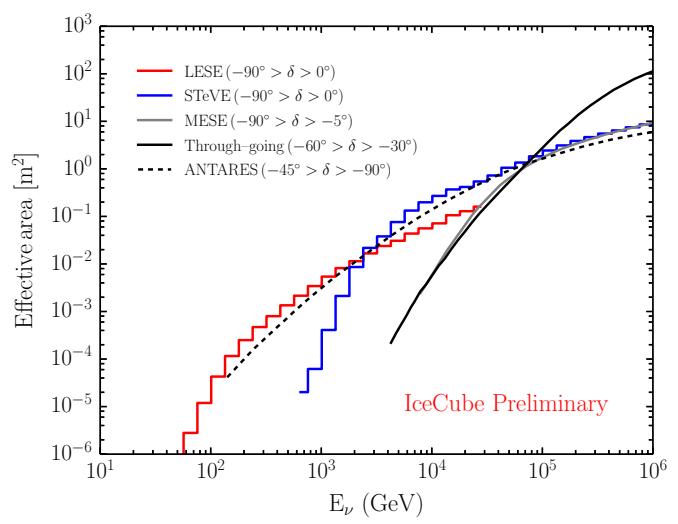

Figure 2. Effective areas of the analyses shown in Fig. 1 and of a corresponding ANTARES analysis [7].

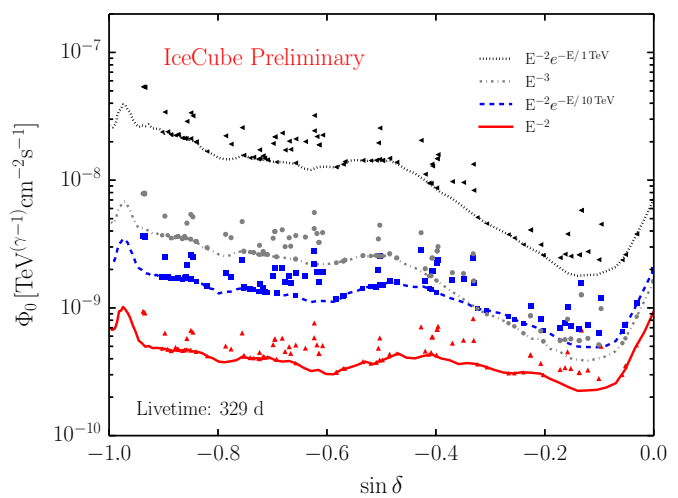

Figure 3. Median sensitivities as a function of declination (lines) and source upper limits (markers) at 90\% C.L. obtained with the LESE analysis for various energy spectra.

depends only on reconstructed declination $\delta$ and reconstructed energy $E$. The likelihood is the product over all $N$ events. It is maximized with the number of signal events $n_{s}$ and the spectral index $\gamma$ as free parameters. The test statistic is then given by the ratio of the likelihood for the null hypothesis $\left(n_{s}=0\right)$ and that for the best fit values.

When testing many points in the sky, trial factors need to be taken into account. To convert the pretrial p-values obtained directly from the test statistic distribution into post-trial p-values, we generate an ensemble of 10000 pseudo-experiments with the R.A. directions scrambled, and count the fraction of these background-only experiments that yield an equal or greater pre-trial p-value.

\section{Results}

Both analyses evaluate the test statistic for all 84 Southern Hemisphere sources in the TeVCat [9] catalog (the "Default" and "Newly announced" catalogs, as of May 2015). We also test 12 sources not in TeVCat, but included in earlier IceCube point source searches. After trial factor correction, none of these 96 sources show a significant over-fluctuation. The obtained $90 \%$ upper limits on the neutrino flux, calculated using the Neyman method [10], are shown in Figs. 3 and 4 together with the sensitivities as a function of declination. Figure 3 shows the LESE results for a few different spectral hypotheses of the 


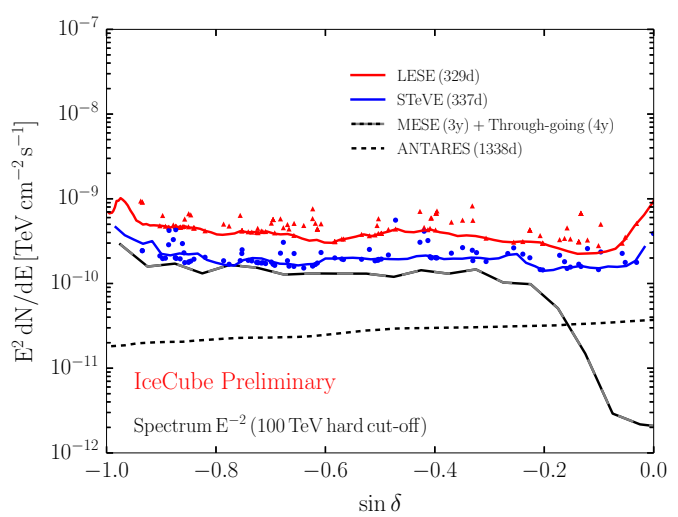

Figure 4. Median sensitivities and source upper limits for an $E^{-2}$ spectrum with a hard cut-off at $100 \mathrm{TeV}$ for LESE and STeVE, compared to a combination of MESE [6] and the standard point source search [1], as well as ANTARES [11].
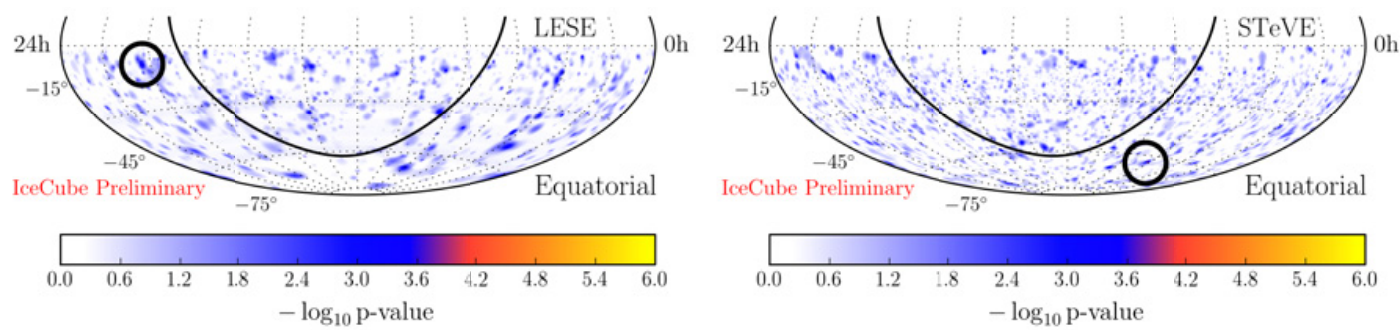

Figure 5. Sky maps of the pre-trial p-values obtained with LESE (left) and STeVE (right).

form $d N / d E=\Phi_{0}(E / \mathrm{TeV})^{-\gamma} \exp \left(-E / E_{\text {cut }}\right)$ with $\gamma=(2,3)$ and $E_{\text {cut }}=(1 \mathrm{TeV}, 10 \mathrm{TeV}, \infty)$. Figure 4 shows limits and sensitivities of both LESE and STeVE for an $E^{-2}$ spectrum with a hard cut-off at $100 \mathrm{TeV}$, compared to the sensitivities of the combined MESE [6] and standard point source analysis [1], and a corresponding ANTARES analysis using 6 years of data [11]. The most significant source in the LESE analysis is QSO 2022-077 (also known as 2EG J2023-0836) at R.A. 306.5 and Dec. -7.6 ${ }^{\circ}$, with a post-trial p-value of $14.8 \%$. For STeVE, the most significant source is SNR G000.9+00.1 at R.A. $266.8^{\circ}$ and Dec. $-28.2^{\circ}$, with a post-trial p-value of $35.4 \%$.

In addition to the pre-defined source list, both analyses perform a scan of the whole Southern Sky on a HEALPix [12] grid with a bin width of about $0.5^{\circ}$, well below the angular resolution for both samples. For each bin, the test statistic is evaluated in the same way as for the source list. The resulting pre-trial sky maps are shown in Fig. 5 for LESE (left) and STeVE (right). Again, no significant clustering of events is found. The hottest spot is marked with a circle in both maps. In the case of LESE, it is found at R.A. $305.2^{\circ}$ and Dec. $-8.5^{\circ}$, close to the most significant source, with a post-trial p-value of $88.1 \%$. The STeVE analysis finds the hottest spot at R.A. $93.1^{\circ}$ and Dec. $-64.3^{\circ}$, with a post-trial p-value of $74.9 \%$ In both maps, the black line marks the Galactic plane.

\section{Summary and outlook}

Complementary to the standard IceCube point source search in the Southern Hemisphere, which is mainly sensitive to neutrinos with energies above $100 \mathrm{TeV}$, we have performed two analyses searching for neutrino point sources at energies as low as $100 \mathrm{GeV}$. The rejection of the large background of 
atmospheric muons is possible by making extensive use of veto information from the outer layers of IceCube. The data are analyzed using the same unbinned likelihood method as the standard point source analysis. No significant neutrino emission has been found. The obtained upper limits are about one order of magnitude weaker than those by ANTARES. Both analyses presented here use data from the first year of the completed IceCube detector with 86 strings. Three more years of data are available and ready to be incorporated. In addition, searches are planned for neutrino emission from extended sources and in particular from the Galactic plane, using the same data samples. In the long term, a unification of the different point-source searches will improve the overall sensitivity.

\section{References}

[1] M.G. Aartsen et al., Astrophys. J. 796(2), 109 (2014), http://stacks.iop.org/0004-637X/ $796 / i=2 / a=109$

[2] M.D. Kistler, J.F. Beacom, Phys. Rev. D 74, 063007 (2006), http://link.aps.org/doi/ 10.1103/PhysRevD.74.063007

[3] A. Kappes, J. Hinton, C. Stegmann, F.A. Aharonian, Astrophys. J. 656(2), 870 (2007)

[4] A. Achterberg et al., Astroparticle Physics 26(3), 155 (2006), ISSN 0927-6505, http:// www.sciencedirect.com/science/article/pii/S0927650506000855

[5] S. Euler, Ph.D. thesis, RWTH Aachen University (2014), http://publications.rwthaachen.de/record/444944

[6] J. Feintzeig, Ph.D. thesis, University of Wisconsin, Madison (2014), http://search . proquest.com/docview/1641120849

[7] S. Adriàąn-Martìnez et al., The Astrophysical Journal 760(1), 53 (2012), http:// stacks. iop.org $/ 0004-637 \mathrm{X} / 760 / i=1 / a=53$

[8] J. Braun et al., Astropart.Phys. 29(4), 299 (2008), ISSN 0927-6505, http://www . sciencedirect.com/science/article/pii/S0927650508000303

[9] S.P. Wakely, D. Horan, TeVCat: An online catalog for Very High Energy Gamma-Ray Astronomy, in Proceedings of the 30th International Cosmic Ray Conference (2007), Vol. 3, p. 1341

[10] J. Neyman, Philosophical Transactions of the Royal Society of London. Series A, Mathematical and Physical Sciences 236(767), 333 (1937), ISSN 00804614, http: //www.jstor.org/stable/91337

[11] S. Adriàąn-Martìnez et al., The Astrophysical Journal Letters 786(1), L5 (2014), http://stacks.iop.org/2041-8205/786/i=1/a=L5

[12] K.M. Gòrski et al., Astrophys. J. 622(2), 759 (2005), http://stacks.iop.org/ $0004-637 \mathrm{X} / 622 / \mathrm{i}=2 / \mathrm{a}=759$ 